\title{
High Tongue Position is a Risk Factor for Upper Airway Concentric Collapse in Obstructive Sleep Apnea: Observation Through Sleep Endoscopy
}

This article was published in the following Dove Press journal: Nature and Science of Sleep

\author{
Chen Zhao (iD) ${ }^{1,2}$ \\ Alonço Viana $\left(\mathbb{D}^{2-4}\right.$ \\ Yifei $\mathrm{Ma}^{5}$ \\ Robson Capasso ${ }^{2}$
}

'Department of Otorhinolaryngology, The First Affiliated Hospital of China Medical University, Shenyang, Liaoning, People's Republic of China; ${ }^{2}$ Division of Sleep Surgery, Department of Otolaryngology- Head \& Neck Surgery, Stanford University Medical Center, Stanford, CA, USA; ${ }^{3}$ Graduate Program of Neurology, Rio de Janeiro State Federal University (UNIRIO), Rio de Janeiro, Brazil; ${ }^{4}$ Department of Otorhinolaryngology, Marcílio Dias Naval Hospital, Rio de Janeiro, Brazil; ${ }^{5}$ Department of Otolaryngology- Head \& Neck Surgery, Stanford University Medical Center, Stanford, CA, USA
Correspondence: Chen Zhao

Department of Otorhinolaryngology, The First Affiliated Hospital of China Medical University, No. 155, North Nanjing Street, Heping District, Shenyang, Liaoning I 1000 I, People's Republic of China

Tel +86 I3998856576

$\mathrm{Fax}+8602483282997$

Email zhaochen@cmu.edu.cn
Purpose: Identification of upper airway (UA) obstruction based on pharyngeal factors is important for obstructive sleep apnea (OSA) evaluation. This study is to assess the association between UA collapse characteristics and Friedman tongue position (FTP) in patients with OSA through drug-induced sleep endoscopy (DISE).

Patients and Methods: Retrospective study in individuals with OSA who were intolerant to continuous positive airway pressure (CPAP) treatment, submitted to DISE between June 1, 2013, and July 31, 2017. All subjects were classified as having an FTP grade of I to IV, and the velum, oropharynx, tongue base, epiglottis (VOTE) classification was used to analyze the DISE findings. UA collapse characteristics by DISE and FTP grading were compared between groups. The associations between specific DISE findings and FTP were analyzed. Results: In total, 205 patients were assessed. A positive and significant correlation was identified between the presence of retropalatal complete concentric collapse (CCC) and FTP grade, according to the following distributions: I, 17.4\%; II, 22.9\%; III, 33.7\%; and IV, $48.7 \%(P=0.014)$. A logistic regression model revealed that CCC was associated with FTP grade IV. After adjusting for age, sex, body mass index (BMI), and tonsil size (TS), the grade IV individuals had a 4.4-fold higher risk of having CCC than grade I individuals $(P=0.026)$. Multiple collapse sites and palatopharyngeal or combined (palatopharyngeal and hypopharyngeal) collapse were more prevalent in grade IV individuals.

Conclusion: OSA patients intolerant to CPAP have a strong positive correlation between the FTP grade and presence of retropalatal CCC. FTP grade IV is an independent risk factor for velum-CCC, controlling for sex, age, BMI, and TS grade.

Keywords: drug-induced sedation endoscopy, sleep endoscopy, obstructive sleep apnea, tongue position, upper airway collapse, complete concentric collapse

\section{Introduction}

Obstructive sleep apnea (OSA) is a common sleep disorder with a prevalence of $2 \%$ to $37 \%$ and $4 \%$ to $50 \%$, respectively, in males and females, based on a review of 11 epidemiological studies published between 1993 and 2013, effectively showing that its prevalence has been rising in recent years. ${ }^{1}$ OSA patients present typical symptoms and morbidities such as daytime sleepiness, nocturnal snoring, wheezing, increased breathing effort, frequent awakenings, and intermittent hypoxemia that compromise their sleep quality, alongside cognitive and behavioral impairment, as well as increased mortality. ${ }^{2,3}$

Upper airway (UA) obstruction during sleep is the main pathogenic mechanism underlying OSA. Several factors contribute to airway collapse susceptibility, such 
as age, gender, body mass index (BMI), neck and waist circumference, craniofacial development and pharyngeal cavity anatomy. ${ }^{4}$ Among the structures that may contribute to this obstruction, the tongue is one of the most important for pharyngeal collapse, due to its increased volume, a compromising tonus, and its interaction with surrounding tissues. By cephalometric analysis, the tongue was found to contribute to velopharyngeal obstruction, as a higher tongue base resulted in increased contact distance with the soft palate; and in hypopharyngeal obstruction, as a more inferior hyoid resulted in an upright tongue position with more tongue tissue occupying the airway. ${ }^{5}$ Based on the relative tongue position, it is possible to predict risk and severity of OSA and sites of UA obstruction. ${ }^{6-8}$ A higher tongue position has already been associated with a higher apnea-hypopnea index (AHI), markedly severe hypopharyngeal obstruction, ${ }^{9}$ and a lower surgical success rate of uvulopalatopharyngoplasty (UPPP). ${ }^{10,11}$

Two classification systems have already addressed the assessment of tongue height based on physical examination of the oral cavity, but not on tongue size. The first system is the modified Mallampati classification (MMC). Initially described as the Mallampati classification, ${ }^{12}$ its score varies from class I to III based on the visualization of the uvula, tonsils, pillars and soft palate with tongue projection. In 1987, Samsoon and Young proposed a modification of the scoring system by adding a class IV, allowing for more details in describing the palate position in relation to the tongue. ${ }^{13}$ This classification has been used as a clinical predictor of successful intubation in anesthesiology. ${ }^{8}$ The second classification system is the Friedman tongue position (FTP) grade, reported by Friedman in 1999. FTP allows for the description of the tongue's natural position on the mouth floor and yields a score varying from I to IV, similar to the MMC. ${ }^{14}$ Both have proven useful in assessing those with OSA. ${ }^{15}$ However, the FTP better reflects OSA severity than the $\mathrm{MMC},{ }^{16}$ and it is considered a classic indicator of OSA, while effectively informing surgical selection. ${ }^{17}$

Although many studies have identified a strong association between an increased OSA incidence and severity and an increased score in the MMP/FTP classifications, ${ }^{9-11,18}$ the clinical features correlated with FTP grades remain diverse. Harvey et al showed that individuals with high FTP scores presented with overall UA volume reduction, and not only a narrowing of the retroglossal area by computed tomography. ${ }^{19}$ A relatively high tongue position was found to be associated with increases in soft palate length and UA obstruction. ${ }^{20}$ In addition, some studies revealed no relationship between increasing FTP and UA obstruction..$^{21,22}$

Drug-induced sleep endoscopy (DISE) is a tool widely used to evaluate UA collapse in OSA individuals in recent years, despite some well-known limitations. ${ }^{23}$ It enables a dynamic and consecutive observation of the airway lumen during drug-induced sleep, mimicking natural sleep. Different collapse patterns have enabled accurate surgery selection and management. ${ }^{24}$ These DISE findings may help explain the contradictions in the literature regarding FTP grade and OSA severity.

In this context, this study aimed to identify the characteristics of UA collapse in relation to FTP grade, especially in regard to high grades, in OSA individuals.

\section{Patients and Methods Study Design}

This was a retrospective study that analyzed all DISE exams performed on OSA patients at the Stanford Sleep Surgery Clinic between June 1, 2013, and July 31, 2017, and was approved by the Institutional Review Board and Hospital Research Ethics Committee of Stanford Hospital and Clinics (Protocol 35054).

Individuals with an apnea-hypopnea index (AHI) $\geq 5$ events/hour, recorded by overnight polysomnography (PSG) in the laboratory or through a home sleep test (HST), and also with continuous positive airway pressure (CPAP) intolerance, were included in the study. Individuals with a previous invasive treatment for OSA, such as UPPP, tongue base surgery (transoral robotic surgery, radiofrequency, or coblation), genioglossus advancement, or maxillomandibular advancement, were excluded.

The sample characteristics, including age, sex, body mass index (BMI), tonsil size (TS) grading, FTP grading, Epworth Sleepiness Scale (ESS) score, sleep study data such as AHI and oxygen desaturation index (ODI), lowest oxygen saturation (LSAT), and DISE findings were extracted from the Stanford Hospitals and Clinics electronic health record system (REDCap [Research Electronic Data Capture] and Epic) databases. The above-mentioned clinical features were compared between groups. The associations of UA collapse characteristics (sites, patterns and degree) and FTP grades I to IV were analyzed.

\section{Sleep Studies}

All patients underwent overnight PSG or HST according to the American Academy of Sleep Medicine 2012 edition. ${ }^{25}$ 
Apnea was identified when the airflow amplitude decreased by $\geq 90 \%$ for $\geq 10$ seconds. Hypopnea was identified when airflow amplitude decreased by $\geq 30 \%$ for $\geq 10$ seconds and was associated with an oxygen desaturation increase by $\geq 3 \%$ or arousal. AHI represented the number of apnea and hypopnea events per hour. ODI represented the number of times per hour that oxygen desaturation increased by $\geq 3 \%$. LSAT represented the lowest $\mathrm{O}_{2}$ saturation during sleep.

\section{Friedman Tongue Position and Tonsil Size Grading}

FTP grading was performed with an open mouth and the tongue resting on the mouth floor and evaluated with a minimum of 5 times. Individuals included in the study were subdivided into the following four groups: FTP I, uvula and tonsils completely visible; FTP II, visible uvula but not tonsils; FTP III, visible soft palate, but not uvula; FTP IV, visible hard palate only. Tonsil size was graded accordingly: 0 , tonsils absent; 1 , tonsils hidden inside the pillars; 2 , tonsils extending to the pillars; 3 , tonsils extending beyond the pillars; 4, tonsils extending to the midline. ${ }^{10}$ All evaluations were performed and graded by the same senior and experienced surgeon (R.C).

\section{Drug-Induced Sleep Endoscopy}

DISE was performed in the operating room in a supine position. Dexmedetomidine was used for sedation in two stages, with an initial intravenous bolus of $1.5 \mathrm{mcg} / \mathrm{kg}$ over 10 minutes, followed by a continuous infusion at $1.5 \mathrm{mcg} / \mathrm{kg} / \mathrm{h}$. After two consecutive cycles of snoring and stopping breathing, a flexible endoscope was inserted through the nostril and positioned in the retropalatal and retrolingual regions. The minimum time for the exam recordings was 20 minutes, reducing the chances of collapse by over-induction. The Velum-Oropharynx-Tongue base-Epiglottis (VOTE) classification was used to describe the DISE findings, according to the following characteristics: sites (velum [V], oropharynx [O], tongue base [T], and epiglottis [E]); pattern (anteroposterior [AP], lateral [L], or concentric [C]); and degree of collapse (absent [0], partial [1], or complete [2]). ${ }^{26}$ The VOTE obstruction sites were also analyzed as palatopharynx (higher), velum, and/ or oropharynx; hypopharynx (lower), tongue base, and/or epiglottis; and combined, in both cases. All DISE exams were performed and classified by the same senior and experienced surgeon (R.C).

\section{Statistical Analysis}

Normally distributed continuous variables were represented as mean and standard deviation (SD) and compared between FTP classification groups using ANOVA. Non-normally distributed variables were represented as median (range) and compared using a Wilcoxon Rank Sum test. Categorical variables were presented as frequencies (percentages, \%) and compared using a Fisher's exact test or Wilcoxon Rank Sum test if categories were ordered. Logistical regression model was used with FTP grades as the main predictor and binary collapse characteristics, including degree (no, partial, complete) and pattern (AP, L, C) at sites $\mathrm{V}, \mathrm{O}$, $\mathrm{T}$ and $\mathrm{E}$ as outcome variables. The odds ratio (OR) of collapse associated with FTP grades II, III, and IV in reference to FTP grade I were then estimated without and with adjustment for age, gender, BMI, and tonsil size. Differences were considered statistically significant if $P<0.05$. Statistical analyses were performed using SAS 9.4 (SAS Inc., Cary, NC, USA).

\section{Results}

Among 225 records found, 20 were excluded, most commonly because these individuals had undergone a previous UA surgery. The samples (205) were allocated to FTP I $(\mathrm{n}=23)$, FTP II $(\mathrm{n}=48)$, FTP III $(\mathrm{n}=95)$, and FTP IV $(\mathrm{n}=39)$ groups based on the FTP classification, and no significant differences were found in the analyzed baseline characteristics (except for age) including gender, BMI, ESS, AHI, ODI, LSAT, and tonsil size (Table 1).

The presence of complete collapse in more than one site was the most common finding, highlighting the involvement of combined palatopharyngeal and hypopharyngeal collapse and isolated palatopharyngeal collapse. Isolated collapse of the hypopharyngeal sites ( $\mathrm{T}$ and $\mathrm{E}$ ) was the least commonly seen among the groups. No significant differences were identified between collapse sites and different FTP grades (Table 2).

The collapse patterns and degrees at the various sites, across different FTP grades, are shown in Table 3. The presence of velum complete concentric collapse (CCC) increased significantly among groups as the FTP grade increased: $17.4 \%, 22.9 \%, 33.7 \%$, and $48.7 \%$, respectively, for FTP grades I to IV $(P=0.014)$. Collapse differences at the tongue base and other sites between groups were not significantly different $(P>0.05)$. 
Table I Sample Baseline Characteristics According to Different FTP Grades

\begin{tabular}{|c|c|c|c|c|c|}
\hline Characteristics & FTP I $(n=23)$ & FTP II $(n=48)$ & FTP III $(n=95)$ & FTP IV $(n=39)$ & $P$-value \\
\hline Age $(y)^{a}$ & $43.57 \pm 13.85$ & $51.52 \pm 15.87$ & $50.83 \pm 14.08$ & $42.28 \pm 16.28$ & 0.04 \\
\hline Male $^{\mathrm{b}}$ & $20(87 \%)$ & 34 (70.8\%) & 79 (83.2\%) & 30 (76.9\%) & 0.268 \\
\hline BMI $\left(\mathrm{kg} / \mathrm{m}^{2}\right)^{\mathrm{a}}$ & $27.17 \pm 4.57$ & $26.74 \pm 4.21$ & $28.46 \pm 4.21$ & $28.84 \pm 5.55$ & 0.082 \\
\hline ESS $^{\mathrm{a}}$ & $8.62 \pm 5.02$ & $9.09 \pm 5.89$ & $10.2 \pm 5.07$ & $9.89 \pm 4.67$ & \\
\hline c & $8(5-13)$ & $8(4-14)$ & $10(6-14)$ & $10(7-13)$ & 0.585 \\
\hline $\mathrm{AHI}$ (events/h) ${ }^{\mathrm{a}}$ & $30.87 \pm 19.4$ & $34.54 \pm 21.29$ & $38.04 \pm 20.13$ & $38.4 I \pm 22.13$ & \\
\hline c & $28(17.4-42.1)$ & $30.9(19.1-45.5)$ & $34.5(21.9-51.3)$ & $39.5(19.8-53.6)$ & 0.396 \\
\hline ODI (events/h) ${ }^{a}$ & $22.89 \pm 23.36$ & $20.87 \pm 16.39$ & $27.09 \pm 23.37$ & $25.18 \pm 22.43$ & \\
\hline c & I5.4 (3.15-32.65) & $15.15(10.5-30)$ & $20.5(10-41.3)$ & $19.75(8.85-31.15)$ & 0.647 \\
\hline LSAT (\%) ${ }^{a}$ & $84.02 \pm 9.86$ & $84.44 \pm 5.92$ & $82.28 \pm 8.89$ & $84 \pm 7.9$ & \\
\hline c & $87(82-90)$ & $85.5(81-89)$ & 84 (79-89) & $87(78-90)$ & 0.419 \\
\hline \multicolumn{6}{|l|}{ TS $^{b}$} \\
\hline Grade 0 & $3(13 \%)$ & $8(16.7 \%)$ & $19(20 \%)$ & $8(20.5 \%)$ & \\
\hline Grade I & $14(60.9 \%)$ & $28(58.3 \%)$ & 47 (49.5\%) & 15 (38.5\%) & \\
\hline Grade 2 & $4(17.4 \%)$ & $10(20.8 \%)$ & $25(26.3 \%)$ & $9(23.1 \%)$ & \\
\hline Grade 3 & $2(8.7 \%)$ & $2(4.2 \%)$ & $4(4.2 \%)$ & $5(12.8 \%)$ & \\
\hline Grade 4 & $0(0 \%)$ & $0(0 \%)$ & $0(0 \%)$ & $2(5.1 \%)$ & 0.19 \\
\hline
\end{tabular}

Notes: a Mean \pm Standard Deviation; ${ }^{b} \mathrm{n}(\%) ;{ }^{\mathrm{c} M e d i a n}$ (Range).

Abbreviations: FTP, Friedman tongue position; I, uvula and tonsils completely visible; II, visible uvula and not tonsils; III, visible soft palate, but not uvula; IV, visible only hard palate; BMI, body mass index; ESS, Epworth Sleepiness Scale; AHI, Apnea Hypopnea Index; ODI, Oxygen Desaturation Index; LSAT, lowest oxygen saturation; TS, tonsil size; grade 0 , absent; grade I, behind tonsillar pillars; grade 2, extend to pillars; grade 3, visible beyond pillars; grade 4, enlarged to midline.

FTP grade IV was found to be significantly associated with an increased velum-CCC by a logistic regression model, compared to the FTP I group, persisting after adjustment for age, gender, BMI, and tonsil size (Table 4). The FTP IV group was about 4.5-fold more likely to have a velum-CCC than the FTP I group $(P=0.026)$ by an OR analysis. Other collapse characteristics were not associated with the FTP grades $(P>0.05)$.

\section{Discussion}

In this study, we identified greater proportions of retropalatal CCC in OSA patients with a higher FTP grade. FTP grade IV was an independent risk factor for velum-CCC, controlling for age, gender, BMI, and tonsil size. To date, we have not identified, in previously published literature, any association between a high tongue position, reflected in FTP grade, and a concentric collapse pattern found on DISE.

Table 2 Partial Collapse and Sites of Complete Collapse Among Different FTP Grades

\begin{tabular}{|l|l|l|l|l|l|}
\hline Collapse & FTP I (n=23) & FTP II (n=48) & FTP III (n=95) & FTP IV (n=39) & P-value \\
\hline PARTIAL & $5(21.7 \%)$ & $3(6.3 \%)$ & $5(5.3 \%)$ & $2(5.1 \%)$ \\
\hline $\begin{array}{l}\text { COMPLETE } \\
\text { Sum of sites }\end{array}$ & & & & \\
I & $2(8.7 \%)$ & $20(41.7 \%)$ & $29(30.5 \%)$ & $9(23.1 \%)$ \\
2 & $12(52.2 \%)$ & $16(33.3 \%)$ & $45(47.4 \%)$ & $21(53.8 \%)$ \\
$\geqq 3$ & $4(17.4)$ & $9(18.8 \%)$ & $16(16.8 \%)$ & $7(17.9 \%)$ \\
\hline Sites & & & $46(48.4 \%)$ & $21(53.8 \%)$ \\
Palatopharynx & $7(30.4 \%)$ & $19(39.6 \%)$ & $6(6.3 \%)$ & $2(5.1 \%)$ \\
Hypopharynx & $3(13 \%)$ & $6(12.5 \%)$ & $38(40 \%)$ & $14(35.9 \%)$ \\
Combined & $8(34.8 \%)$ & $20(41.7 \%)$ & 0.078 \\
\hline
\end{tabular}

Notes: Partial collapse: $50 \%$ to $75 \%$ lumen reduction; complete collapse: $75 \%$ to $100 \%$ lumen reduction. Sum of sites: I, one site showed complete collapse; 2 , two sites showed complete collapse; $\geqq 3$, three or four sites showed complete collapse. Palatopharynx, velum and/or oropharynx sites; hypopharynx, tongue base and/or epiglottis sites; combined, palatopharynx and hypopharynx.

Abbreviations: FTP, Friedman tongue position; I, uvula and tonsils completely visible; II, visible uvula but not tonsils; III, visible soft palate, but not uvula; IV, visible hard palate only. 
Table 3 Collapse Pattern and Degree Among Different FTP Grades

\begin{tabular}{|c|c|c|c|c|c|c|c|}
\hline \multirow[t]{3}{*}{ Site } & \multirow[t]{3}{*}{ Pattern } & \multirow[t]{3}{*}{ Degree } & FTP I & FTP II & FTP III & FTP IV & \multirow[t]{3}{*}{$P$-value } \\
\hline & & & $(n=23)$ & $(n=48)$ & $(n=95)$ & $(n=39)$ & \\
\hline & & & n (\%) & n (\%) & n (\%) & n (\%) & \\
\hline \multirow[t]{9}{*}{ V } & AP & 0 & $\mathrm{I}(4.3 \%)$ & $5(10.4 \%)$ & $3(3.2 \%)$ & $2(5.1 \%)$ & \\
\hline & & I & $4(17.4 \%)$ & $4(8.3 \%)$ & $7(7.4 \%)$ & $0(0 \%)$ & \\
\hline & & 2 & $8(34.8 \%)$ & $25(52.1 \%)$ & $47(49.5 \%)$ & $16(41 \%)$ & 0.144 \\
\hline & L & 0 & $0(0 \%)$ & $0(0 \%)$ & $0(0 \%)$ & $0(0 \%)$ & \\
\hline & & I & $\mathrm{I}(4.3 \%)$ & $0(0 \%)$ & $\mathrm{I}(\mathrm{I} .1 \%)$ & $0(0 \%)$ & \\
\hline & & 2 & $2(8.7 \%)$ & $0(0 \%)$ & $4(4.2 \%)$ & $0(0 \%)$ & 0.143 \\
\hline & C & 0 & $0(0 \%)$ & $0(0 \%)$ & $0(0 \%)$ & $\mathrm{I}(2.6 \%)$ & \\
\hline & & I & $3(13 \%)$ & $3(6.3 \%)$ & $\mathrm{I}(\mathrm{I} .1 \%)$ & $I(2.6 \%)$ & \\
\hline & & 2 & $4(17.4 \%)$ & II (22.9\%) & $32(33.7 \%)$ & $19(48.7 \%)$ & 0.014 \\
\hline \multirow[t]{3}{*}{ O } & L & 0 & $8(34.8 \%)$ & $19(39.6 \%)$ & $25(26.3 \%)$ & $8(20.5 \%)$ & \\
\hline & & I & $7(30.4 \%)$ & $19(39.6 \%)$ & $38(40 \%)$ & $10(25.6 \%)$ & \\
\hline & & 2 & $8(34.8 \%)$ & $10(20.8 \%)$ & $32(33.7 \%)$ & $2 \mathrm{I}(53.8 \%)$ & 0.061 \\
\hline \multirow[t]{3}{*}{$\mathrm{T}$} & AP & 0 & $6(26.1 \%)$ & I5(3|.3\%) & $22(23.2 \%)$ & $9(23.1 \%)$ & \\
\hline & & I & $7(30.4 \%)$ & I3(27.1\%) & $43(45.3 \%)$ & $19(48.7 \%)$ & \\
\hline & & 2 & $10(43.5 \%)$ & $20(4 \mid .7 \%)$ & $30(31.6 \%)$ & II(28.2\%) & 0.342 \\
\hline \multirow[t]{6}{*}{$E$} & $\mathrm{AP}$ & 0 & $16(69.6 \%)$ & $33(68.8 \%)$ & $6 \mathrm{I}(64.2 \%)$ & $26(66.7 \%)$ & \\
\hline & & I & $0(0 \%)$ & $2(4.2 \%)$ & $9(9.5 \%)$ & $6(15.4 \%)$ & \\
\hline & & 2 & $6(26.1 \%)$ & II (22.9\%) & $24(25.3 \%)$ & $6(15.4 \%)$ & \\
\hline & L & 0 & $0(0 \%)$ & $0(0 \%)$ & $0(0 \%)$ & $0(0 \%)$ & \\
\hline & & I & $0(0 \%)$ & $\mathrm{I}(2.1 \%)$ & $0(0 \%)$ & $\mathrm{I}(2.6 \%)$ & \\
\hline & & 2 & $\mathrm{I}(4.3 \%)$ & $I(2.1 \%)$ & $I(I . I \%)$ & $0(0 \%)$ & 0.337 \\
\hline
\end{tabular}

Notes: 0 , no collapse with $0 \%$ to $50 \%$ lumen reduction; I, partial collapse with $50 \%$ to $75 \%$ lumen reduction; 2 , complete collapse with $75 \%$ to $100 \%$ lumen reduction. Abbreviations: FTP, Friedman tongue position; I, uvula and tonsils completely visible; II, visible uvula but not tonsils; III, visible soft palate, but not uvula; IV, visible hard palate only; V, velum; O, oropharynx; T, tongue base; E, epiglottis; AP, anterior-posterior; L, lateral; C, concentric.

In this study, the overall proportion of velum-CCC was $31.2 \%$, consistent with that of large-sample studies that reported $31.5 \%$ and $37.3 \%$ in 1249 and 424 individuals with OSA, respectively. ${ }^{27,28}$ This collapse pattern increased from $17.4 \%$ in FTP grade I to $48.7 \%$ in grade IV. In previous studies, an increased CCC at the retropalatal site was found to be associated with obesity. ${ }^{27,29}$ In this study, we found that a CCC increase was associated with a higher FTP grade, especially grade IV. Interactions between UA anatomy and muscle function may contribute to this result. In previous research, a high FTP grade was usually accompanied by a low hyoid position and incurred a higher

Table 4 Association Among FTP Grade IV and Characteristics of Complete Collapse Sites by DISE

\begin{tabular}{|l|l|l|l|l|l|l|}
\hline Site & Pattern & Degree & Unadjusted OR (95\% CI) & P-value & Adjusted OR (95\% CI) & P-value \\
\hline Velum & AP & 2 & $1.3(0.45-3.8)$ & 0.626 & $1.5(0.49-4.59)$ & 0.48 \\
& L & 2 & 0 & 1 & 0 & I \\
& C & 2 & $4.5 I(1.3-15.7 I)$ & 0.018 & $4.4(1.19-16.23)$ & 0.026 \\
\hline Oropharynx & L & 2 & $2.19(0.75-6.34)$ & 0.149 & $2.04(0.65-6.42)$ & 0.223 \\
Tongue base & AP & 2 & $0.51(0.17-1.5)$ & 0.223 & $0.54(0.18-1.64)$ & 0.281 \\
Epiglottis & AP+L & 2 & $0.42(0.12-1.44)$ & 0.166 & $0.48(0.14-1.71)$ & 0.258 \\
\hline
\end{tabular}

Notes: 2, complete collapse with $75 \%$ to $100 \%$ lumen reduction; OR adjusted for age, gender, body mass index, tonsil size grade with FTP I and tonsil grade 0 as reference. Abbreviations: FTP, Friedman tongue position; I, uvula and tonsils completely visible; II, visible uvula but not tonsils; III, visible soft palate, but not uvula; IV, visible hard palate only; DISE, drug-induced sleep endoscopy; AP, anterior-posterior; L, lateral; C, concentric. 
prevalence of CCC, as proven by sleep magnetic resonance imaging in severe OSA patients. ${ }^{19,30}$ Moreover, the lateral muscles of the tongue and soft palate were thought to be coupled. $^{31}$ The circumference pattern may be explained by the increase in magnitude of the tongue drop, incurring pharyngeal occupation and central AP compression and posterior movement of the lateral margins of the soft palate, constituting a circumferential constriction.

As known, the presence of CCC was associated with clinical features in OSA patients, including higher respiratory disturbance index and $\mathrm{AHI},{ }^{32}$ a more severe hypoxemia, ${ }^{33}$ and susceptibility to non-positional OSA. ${ }^{28}$ This collapse pattern can increase CPAP titration pressure requirement and rates of surgical failure, ${ }^{34,35}$ as observed in UPPP and hypoglossal nerve stimulation (HGNS). ${ }^{36-38}$ This association also explains why a high tongue position, especially FTP IV, is a predictor of severe OSA, ${ }^{39,40}$ and causes significant reduction in AHI improvement after HGNS. ${ }^{41}$ In this context, the identification of velum-CCC based on its association with FTP grade, as observed by a simple physical examination, has great clinical relevance in individuals with OSA.

Previous research demonstrated that a high tongue position indicated a more severe hypopharyngeal obstruction on DISE. ${ }^{42}$ However, there was no statistically significant difference in this study, in which a complete tongue base collapse was identified in $30.6 \%$ of individuals with FTP grades III and IV, while Wang et al found an incidence of $32 \%$ in individuals with MMC classes III and IV. $^{42}$ In addition, we found no association between retroglossal collapse and tongue position on DISE, similar to the findings of den Herder et al. ${ }^{43}$ Tongue base collapse has a more complex pathogenesis, related not only to tongue position and/or volume but also to muscle tone during sleep. ${ }^{44,45}$

There were some limitations to this study to consider. (i) Age was significantly different between groups, and the effect of aging on velum-CCC or AP collapse patterns is controversial. $^{46,47}$ Moreover, age was included in the logistic regression model to control for its influence. (ii) As a UA collapsibility evaluation method, DISE itself has some limitations, such as controversy about the reliability of observed obstructive sites, ${ }^{48}$ that it might not improve surgical success rates, ${ }^{49}$ and variable findings with prolonged observation time or different sedative drugs. ${ }^{44,50}$ Dexmedetomidine was used for sedation, and differences in tongue base collapse related to propofol or midazolam use have already been identified. Nevertheless, the velum collapse pattern was similar in response to the three drugs, ${ }^{51}$ enabling extension of the findings in this study to DISE with these drugs. (iii) In the sample, only OSA individuals intolerant to CPAP were evaluated, generating a selection bias, and not fully representing the general OSA population. (iv) The sample number in this study was relatively small, especially for the FTP IV group, probably leading to a low statistical power. More observation will be conducted in the future study.

\section{Conclusion}

This study identified a positive correlation between a higher tongue position and retropalatal concentric collapse in OSA patients. FTP grade IV was an independent risk factor for CCC when controlling for age, gender, $\mathrm{BMI}$, and tonsil size.

\section{Ethics Approval}

All procedures performed in this study were in accordance with the ethical standards of the Institutional Review Board and Hospital Research Ethics Committee of Stanford Hospital and Clinics (Protocol 35,054). Patient informed consent was waived because of the retrospective nature of this research. The patients' data were maintained with confidentiality and in compliance with the Declaration of Helsinki.

\section{Disclosure}

Dr Robson Capasso reports personal fees from and served at the advisory board for Invicta Medical, outside the submitted work. The authors report no other conflicts of interest in this work.

\section{References}

1. Franklin KA, Lindberg E. Obstructive sleep apnea is a common disorder in the population-a review on the epidemiology of sleep apnea. $J$ Thorac Dis. 2015;7(8):1311-1322.

2. Jordan AS, McSharry DG, Malhotra A. Adult obstructive sleep apnoea. Lancet. 2014;383(9918):736-747. doi:10.1016/S01406736(13)60734-5

3. Veasey SC, Rosen IM. Obstructive sleep apnea in adults. $N$ Engl J Med. 2019;380(15):1442-1449. doi:10.1056/NEJMcp1816152

4. Pham LV, Schwartz AR. The pathogenesis of obstructive sleep apnea. J Thorac Dis. 2015;7(8):1358-1372.

5. Lyberg T, Krogstad O, Djupesland G. Cephalometric analysis in patients with obstructive sleep apnoea syndrome: II. Soft tissue morphology. J Laryngol Otol. 1989;103(3):293-297. doi:10.1017/ S0022215100108746

6. Avincsal MO, Dinc ME, Ulusoy S, Dalgic A, Ozdemir C, Develioglu ON. Modified Mallampati score improves specificity of STOP-BANG questionnaire for obstructive sleep apnea. J Craniofac Surg. 2017;28(4):904-908. doi:10.1097/SCS.0000000000003513

7. Barceló X, Mirapeix RM, Bugés J, Cobos A, Domingo C. Oropharyngeal examination to predict sleep apnea severity. Arch Otolaryngol Head Neck Surg. 2011;137(10):990-996. doi:10.1001/ archoto.2011.176 
8. Nuckton TJ, Glidden DV, Browner WS, Claman DM. Physical examination: mallampati score as an independent predictor of obstructive sleep apnea. Sleep. 2006;29(7):903-908. doi:10.1093/sleep/29.7.903

9. Karakoc O, Akcam T, Gerek M, Genc H, Ozgen F. The upper airway evaluation of habitual snorers and obstructive sleep apnea patients. ORL J Otorhinolaryngol Relat Spec. 2012;74(3):136-140. doi:10. 1159/000337134

10. Friedman M, Ibrahim H, Bass L. Clinical staging for sleep-disordered breathing. Otolaryngol Head Neck Surg. 2002;127(1):13-21. doi:10.1067/mhn.2002.126477

11. Choi JH, Lee JY, Cha J, Kim K, Hong SN, Lee SH. Predictive models of objective oropharyngeal OSA surgery outcomes: success rate and AHI reduction ratio. PLoS One. 2017;12(9):e0185201. doi:10.1371/ journal.pone. 0185201

12. Mallampati SR, Gatt SP, Gugino LD, et al. A clinical sign to predict difficult tracheal intubation: a prospective study. Can Anaesth Soc J. 1985;32(4):429-434. doi:10.1007/BF03011357

13. Samsoon GL, Young JR. Difficult tracheal intubation: a retrospective study. Anaesthesia. 1987;42(5):487-490. doi:10.1111/j.1365-2044.1987. tb04039.x

14. Friedman M, Tanyeri H, LaRosa M, et al. Clinical predictors of obstructive sleep apnea. Laryngoscope. 1999;109(12):1901-1907. doi:10.1097/00005537-199912000-00002

15. Yu JL, Rosen I. Utility of the modified Mallampati grade and Friedman tongue position in the assessment of obstructive sleep apnea. J Clin Sleep Med. 2020;16(2):303-308. doi:10.5664/ jcsm. 8188

16. Friedman M, Hamilton C, Samuelson CG, Lundgren ME, Pott T. Diagnostic value of the Friedman tongue position and Mallampati classification for obstructive sleep apnea: a meta-analysis. Otolaryngol Head Neck Surg. 2013;148(4):540-547. doi:10.1177/ 0194599812473413

17. Lin HC, Lai CC, Lin PW, et al. Clinical prediction model for obstructive sleep apnea among adult patients with habitual snoring. Otolaryngol Head Neck Surg. 2019;161(1):178-185. doi:10.1177/ 0194599819839999

18. Pang KP, Rotenberg BW, Tucker Woodson B. Advanced Surgical Techniques in Snoring and Obstructive Sleep Apnea. San Diego, CA: Plural Publishing, Inc; 2013.

19. Harvey R, O’Brien L, Aronovich S, et al. Friedman tongue position and cone beam computed tomography in patients with obstructive sleep apnea. Laryngoscope Investig Otolaryngol. 2017;2(5):320-324. doi:10.1002/lio2.92

20. Lim JS, Lee JW, Han C, Kwon JW. Correlation of soft palate length with velum obstruction and severity of obstructive sleep apnea syndrome. Auris Nasus Larynx. 2018;45(3):499-503. doi:10.1016/j. an1.2017.07.023

21. Banhiran W, Junlapan A, Assanasen P, Chongkolwatana C. Physical predictors for moderate to severe obstructive sleep apnea in snoring patients. Sleep Breath. 2014;18(1):151-158. doi:10.1007/s11325-0130863-y

22. Erdamar B, Suoglu Y, Cuhadaroglu C, Katircioglu S, Guven M. Evaluation of clinical parameters in patients with obstructive sleep apnea and possible correlation with the severity of the disease. Eur Arch Otorhinolaryngol. 2001;258(9):492-495. doi:10.1007/s004050100367

23. De Vito A, Carrasco Llatas M, Ravesloot MJ, et al. European position paper on drug-induced sleep endoscopy: 2017 update. Clin Otolaryngol. 2018;43(6):1541-1552. doi:10.1111/coa.13213

24. Chong KB, De Vito A, Vicini C. Drug-induced sleep endoscopy in treatment options selection. Sleep Med Clin. 2019;14(1):33-40.

25. Berry RB, Budhiraja R, Gottlieb DJ, et al. Rules for scoring respiratory events in sleep: update of the 2007 AASM Manual for the Scoring of Sleep and Associated Events. Deliberations of the Sleep Apnea Definitions Task Force of the American Academy of Sleep Medicine. J Clin Sleep Med. 2012;8(5):597-619. doi:10.5664/ jesm. 2172
26. Kezirian EJ, Hohenhorst W, de Vries N. Drug-induced sleep endoscopy: the VOTE classification. Eur Arch Otorhinolaryngol. 2011;268(8):1233-1236. doi:10.1007/s00405-011-1633-8

27. Vroegop AV, Vanderveken OM, Boudewyns AN, et al. Drug-induced sleep endoscopy in sleep-disordered breathing: report on 1249 cases. Laryngoscope. 2014;124(3):797-802. doi:10.1002/lary.24479

28. Kastoer C, Benoist LBL, Dieltjens M, et al. Comparison of upper airway collapse patterns and its clinical significance: drug-induced sleep endoscopy in patients without obstructive sleep apnea, positional and non-positional obstructive sleep apnea. Sleep Breath. 2018;22(4):939-948. doi:10.1007/s11325-018-1702-y

29. Ravesloot MJL, de Vries N. One hundred consecutive patients undergoing drug-induced sleep endoscopy: results and evaluation. Laryngoscope. 2011;121(12):2710-2716. doi:10.1002/lary.22369

30. Liu SY, Huon LK, Lo MT, et al. Static craniofacial measurements and dynamic airway collapse patterns associated with severe obstructive sleep apnoea: a sleep MRI study. Clin Otolaryngol. 2016;41 (6):700-706. doi:10.1111/coa.12598

31. Heiser C, Edenharter G, Bas M, Wirth M, Hofauer B. Palatoglossus coupling in selective upper airway stimulation. Laryngoscope. 2017;127(10):E378-E383. doi:10.1002/lary.26487

32. Genta PR, Sands SA, Butler JP, et al. Airflow shape is associated with the pharyngeal structure causing OSA. Chest. 2017;152(3):537-546. doi:10.1016/j.chest.2017.06.017

33. Lan MC, Liu SYC, Lan MY, Modi R, Capasso R. Lateral pharyngeal wall collapse associated with hypoxemia in obstructive sleep apnea: pharyngeal wall collapse and hypoxemia. Laryngoscope. 2015;125 (10):2408-2412. doi:10.1002/lary.25126

34. Lan MC, Hsu YB, Lan MY, et al. The predictive value of drug-induced sleep endoscopy for CPAP titration in OSA patients. Sleep Breath. 2018;22(4):949-954.

35. Koutsourelakis I, Safruddin F, Ravesloot M, Zakynthinos S, de Vries N. Surgery for obstructive sleep apnea: sleep endoscopy determinants of outcome. Laryngoscope. 2012;122(11):2587-2591. doi:10.1002/lary.23462

36. Hsu YS, Jacobowitz O. Does sleep endoscopy staging pattern correlate with outcome of advanced palatopharyngoplasty for moderate to severe obstructive sleep apnea? J Clin Sleep Med. 2017;13 (10):1137-1144. doi: $10.5664 /$ jcsm.6756

37. Zhang P, Ye J, Pan C, Sun N, Kang D. The role of obstruction length and height in predicting outcome of velopharyngeal surgery. Otolaryngol Head Neck Surg. 2015;153(1):144-149. doi:10.1177/ 0194599815576719

38. Ong AA, Murphey AW, Nguyen SA, et al. Efficacy of upper airway stimulation on collapse patterns observed during drug-induced sedation endoscopy. Otolaryngol Head Neck Surg. 2016;154(5):970-977. doi:10.1177/0194599816636835

39. Ruangsri S, Jorns TP, Puasiri S, Luecha T, Chaithap C, Sawanyawisuth K. Which oropharyngeal factors are significant risk factors for obstructive sleep apnea? An age-matched study and dentist perspectives. Nat Sci Sleep. 2016;8:215-219. doi:10.2147/NSS.S96450

40. Hukins C. Mallampati class is not useful in the clinical assessment of sleep clinic patients. J Clin Sleep Med. 2010;6(6):545-549. doi: $10.5664 / \mathrm{jcsm} .27987$

41. Xiao R, Trask DK, Kominsky AH. Preoperative predictors of response to hypoglossal nerve stimulation for obstructive sleep apnea. Otolaryngol Head Neck Surg. 2020;162(3):400-407. doi:10.1177/0194599820901499

42. Wang WM, Hsu YB, Lan MY, et al. The relationship between modified Mallampati score, Müller's maneuver and drug-induced sleep endoscopy regarding retrolingual obstruction. Ann Otol Rhinol Laryngol. 2018;127(7):463-469. doi:10.1177/0003489418778302

43. den Herder C, van Tinteren H, de Vries N. Sleep endoscopy versus modified Mallampati score in sleep apnea and snoring. Laryngoscope. 2005;115(4):735-739. doi:10.1097/01.mlg.000016 1347.07881.6d 
44. Capasso R, Rosa T, Tsou DY, et al. Variable findings for drug-induced sleep endoscopy in obstructive sleep apnea with propofol versus dexmedetomidine. Otolaryngol Head Neck Surg. 2016;154(4):765-770. doi:10.1177/0194599815625972

45. Soares D, Folbe AJ, Yoo G, Badr MS, Rowley JA, Lin HS. Druginduced sleep endoscopy vs awake Müller's maneuver in the diagnosis of severe upper airway obstruction. Otolaryngol Head Neck Surg. 2013;148(1):151-156. doi:10.1177/0194599812460505

46. Vicini C, De Vito A, Iannella G, et al. The aging effect on upper airways collapse of patients with obstructive sleep apnea syndrome. Eur Arch Otorhinolaryngol. 2018;275(12):2983-2990. doi:10.1007/ s00405-018-5163-5

47. Zhao C, Viana A Jr, Ma Y, Capasso R. The effect of aging on drug-induced sleep endoscopy findings. Laryngoscope. 2018;128 (11):2644-2650. doi:10.1002/lary.27265
48. Blumen MB, Latournerie V, Bequignon E, Guillere L, Chabolle F. Are the obstruction sites visualized on drug-induced sleep endoscopy reliable? Sleep Breath. 2015;19(3):1021-1026. doi:10.1007/s11325014-1107-5

49. Golbin D, Musgrave B, Succar E, Yaremchuk K. Clinical analysis of drug-induced sleep endoscopy for the OSA patient. Laryngoscope. 2016;126(1):249-253. doi:10.1002/lary.25516

50. Heo SJ, Park CM, Kim JS. Time-dependent changes in the obstruction pattern during drug-induced sleep endoscopy. Am J Otolaryngol. 2014;35(1):42-47. doi:10.1016/j.amjoto.2013.08.017

51. Viana A, Zhao C, Rosa T, et al. The effect of sedating agents on drug-induced sleep endoscopy findings. Laryngoscope. 2019;129 (2):506-513. doi:10.1002/lary.27298

\section{Publish your work in this journal}

Nature and Science of Sleep is an international, peer-reviewed, open access journal covering all aspects of sleep science and sleep medicine, including the neurophysiology and functions of sleep, the genetics of sleep, sleep and society, biological rhythms, dreaming, sleep disorders and therapy, and strategies to optimize healthy sleep.
The manuscript management system is completely online and includes a very quick and fair peer-review system, which is all easy to use. Visit http://www.dovepress.com/testimonials.php to read real quotes from published authors. 